\title{
Recent Progress in the Research of Eosinophilic Esophagitis and Gastroenteritis
}

\author{
Yoshikazu Kinoshita Norihisa Ishimura Naoki Oshima Hironobu Mikami \\ Eiko Okimoto Di Jin Jiao Shunji Ishihara \\ Department of Gastroenterology and Hepatology, Shimane University School of Medicine, Izumo, Japan
}

\section{Key Words}

Food allergy · Eosinophile · Dysphagia · Eotaxin ·

Helicobacter pylori

\begin{abstract}
Eosinophilic esophagitis (EoE) and gastroenteritis are allergic gastrointestinal diseases mainly caused by food allergens. The number of patients with EoE is rapidly increasing in both Western and Asian countries. Basic knowledge of these diseases has mainly come from studies of EoE and Th2 type allergic reactions, including IL-5, IL-13, and IL-15, thymic stromal protein, and eotaxin 3, which are considered to have important roles. For a diagnosis of EoE, endoscopic abnormalities and histological confirmation of dense eosinophile infiltration in the esophageal epithelial layer are important, in addition to identifying dysphagia symptoms. As for eosinophilic gastroenteritis, blood test findings are more useful and the role of an endoscopic examination is reduced. For both diseases, the infection rate of Helicobacter pylori is lower than in healthy controls. Glucocorticoid administration is standard treatment for these diseases, while proton pump inhibitors are frequently effective for EoE.
\end{abstract}

(c) 2016 S. Karger AG, Basel
(C) 2016 S. Karger AG, Basel

$0012-2823 / 16 / 0931-0007 \$ 39.50 / 0$

\section{Introduction}

Eosinophilic gastrointestinal disorders (EGID) occurring in adults consist of 2 types of diseases. One is eosinophilic esophagitis (EoE), which features dense infiltration of eosinophiles only in esophageal mucosa, and the other is eosinophilic gastroenteritis (EGE), caused by pathological eosinophile infiltration in the gastrointestinal wall irrespective of esophageal involvement. The prevalence of EGE has been reported to be higher than that of EoE, while published studies of EGE are also greater in number $[1,2]$. However, the incidence and prevalence of EoE has been rapidly increasing in recent decades, mainly in Western countries $[1,3]$. In addition, that prevalence is becoming recognized in Asian countries as well $[4,5]$. This review summarizes recent progress in research of EGID.

\section{Recent Progress in Basic and Clinical Research of EoE}

\section{Pathogenesis}

EoE is considered to be an allergic disease caused mainly by the ingestion of allergens contained in foods and possibly as air-bone environmental substances. Al-

\section{KARGER}

E-Mail karger@karger.com www.karger.com/dig
Yoshikazu Kinoshita, MD, PhD

Professor and Chairman, Department of Gastroenterology and Hepatology, Shimane University School of Medicine 89-1, Enya, Izumo, Shimane 693-8501 (Japan)

E-Mail kinosita@med.shimane-u.ac.jp 


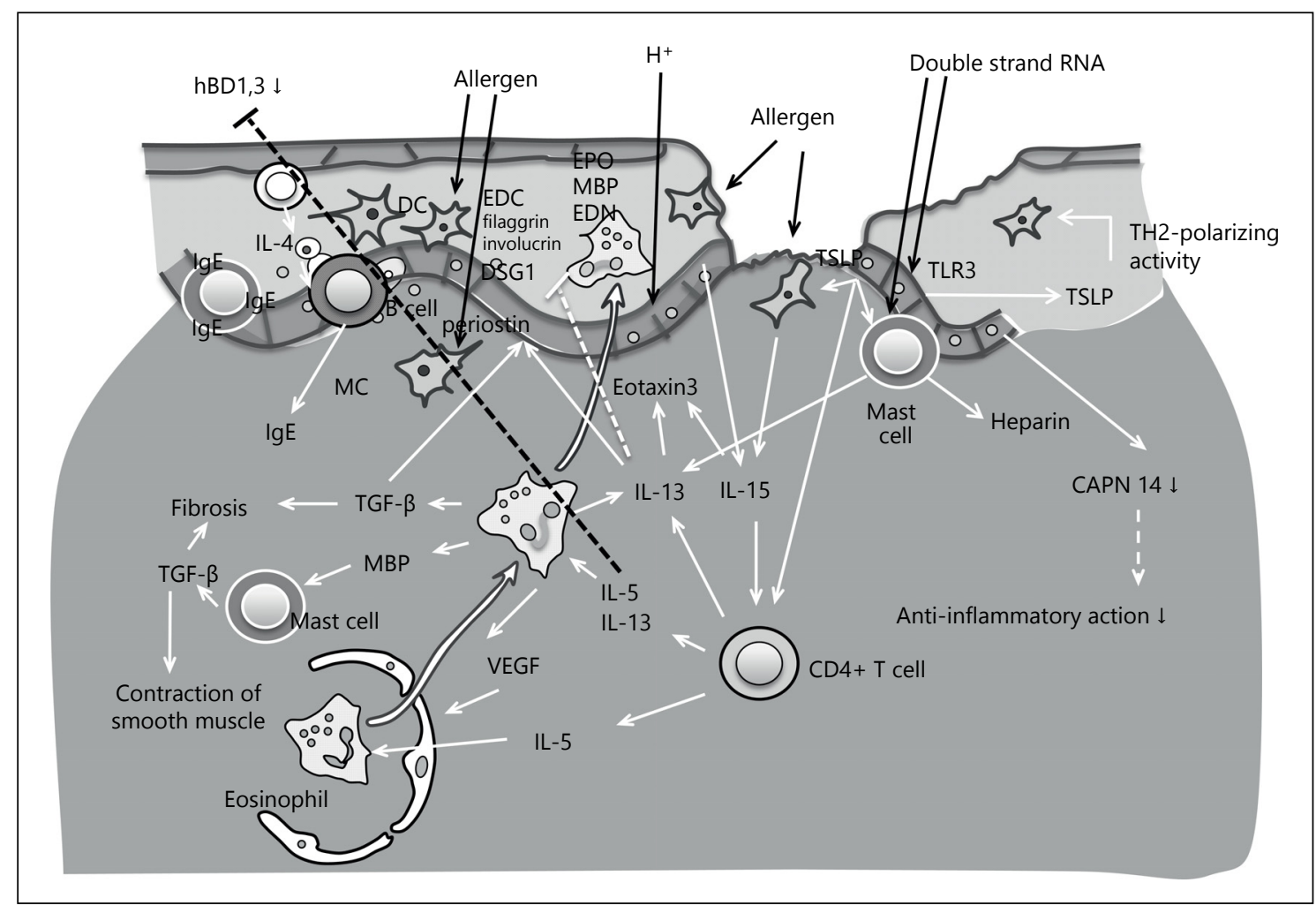

Fig. 1. Schema showing suggested pathogenesis of EoE.

lergenic foods recognized to cause EoE include wheat, egg, milk, soy, nuts, and fish/shell fish. Non-IgE-mediated Th2 responses are considered to be important, with IL-5, IL-13, and IL-15, eotaxin 3, thymic stromal lymphopoietin (TSLP), transforming growth factor- $\beta$, and periostin reported to be major players in disease development (fig. 1) [6]. In general, allergic diseases are increasing in prevalence in developed areas throughout the world. Immune modulation by bacterial and parasitic infections is considered to be critically important for the normal development of the immune system. Thus, a lack of bacterial infection-induced Th1 shift or induction of regulatory $\mathrm{T}$ ( $\mathrm{T}$ reg) cells in the immune system may be important causes of allergic diseases including EGID, which is referred to as the hygiene hypothesis [7].

We recently investigated the prevalence and concentrations of allergen-specific serum IgE in cases with EoE [8], and found that total as well as allergen-specific IgE levels were increased in cases in EoE, though no specific change in IgE-mediated immunity was noted. These results confirmed that EoE is a non- or less-IgE-mediated allergic disease. In addition, the rate of Helicobacter $p y$ lori $(\mathrm{Hp})$ infection was found to be significantly lower in patients with EoE (approximately 20\%) as compared to healthy age- and sex-matched controls (55\%) [9]. A similar lower prevalence of Hp infection was also reported in the United States [10]. In other studies, Hp infection was reported to stimulate Th1 responses, cause a Th1 shift from a Th2 dominant state, induce $\mathrm{T}$ reg cells, and stimulate IL-10 production by $\mathrm{T}$ reg and dendritic cells [11-14]. The decreasing trend of $\mathrm{Hp}$ infection may be one of the causes of increasing incidence of EoE in Western countries and Japan.

\section{Diagnosis}

The mean age of patients with EoE in Japan has been reported to range from 40 to 50 years old, of whom 70$80 \%$ are male [2]. As described above, approximately $80 \%$ of Japanese patients are not infected by $\mathrm{Hp}$, in contrast to an infection rate of $50 \%$ in control cases of similar age [9]. In addition, $50 \%$ of reported patients are affected by allergic diseases including bronchial asthma, atopic dermatitis, allergic rhinitis, and food allergies [2]. Therefore, middle-aged $\mathrm{Hp}$-uninfected males with allergic and atopic diseases are considered to be at the highest risk for development EoE. 


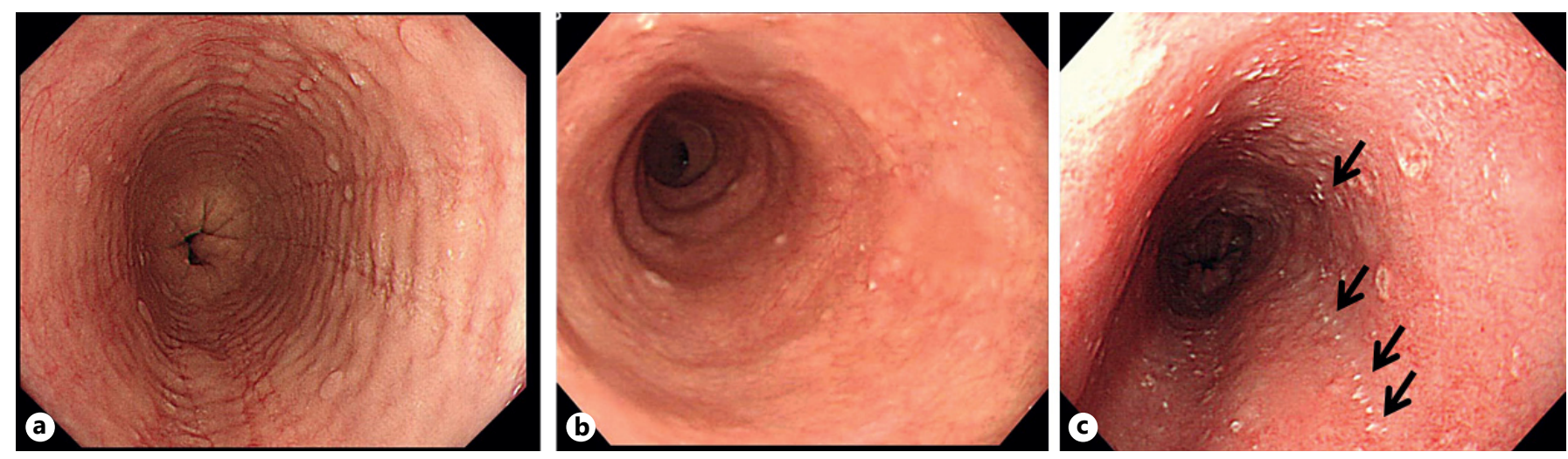

Fig. 2. Characteristic endoscopic features of EoE. Longitudinal furrows and transient concentric rings are shown in (a), fixed concentric rings in (b), and white plaques (arrows) in (c).

For diagnosis of EoE, recognition of characteristic symptoms is considered to be important. Dysphasia is the most frequently reported symptom in patients with EoE, followed by heartburn and chest pain, while food impaction, rarely reported in Asian patients, is one of the specific symptoms noted in those in Western countries. However, our recent study showed that the specificity and sensitivity of symptom recognition for diagnosis are unfortunately lower than expected [15].

Blood tests are neither adequately sensitive nor specific. For example, only $30 \%$ of reported cases have peripheral eosinophilia [2]. Furthermore, though $70 \%$ of the cases have an elevated plasma IgE concentration, that specificity is not adequate [8]. We previously measured the plasma concentrations of IL-5, IL-13, and IL-15, eotaxin 3, and TSLP, and then investigated whether measured levels of those cytokines were helpful for accurate diagnosis of EoE [16]. We concluded that such measurements were not effective because of large overlaps between the EoE patients and normal controls. Recently, Dellon et al. [17] also reported the limited role of cytokine measurement as an additional diagnostic factor.

Therefore, endoscopy is now considered to be the most important diagnostic tool. Longitudinal furrows, fixed and non-fixed concentric rings, white plaques, nodularity, and mucosal edema with decreased mucosal vascular visibility have each been reported to be endoscopic characteristics in patients with EoE. Among these abnormalities, longitudinal furrows are considered to be the most helpful findings followed by concentric rings for diagnosis of EoE, at least in Japanese patients (fig. 2) [15]. When such findings are obtained, multiple sampling of endoscopic biopsy specimens must be performed.
A minimum of 5 biopsy samples are required to increase the diagnostic sensitivity of histo-pathological examinations [18]. Specimens obtained from the distal esophagus may increase sensitivity, since eosinophile density there is reported to be higher [19]. For histological diagnosis, at least 15-20 eosinophiles/high power microscopic fields are considered necessary. Eosinophile degranulation, eosinophile micro-abscesses, fibrosis of the subepithelial layer, and other types of immunocytes including basophiles may also be found in EoE cases.

For clinical diagnosis of EoE, clinico-pathological diagnosis including the presence of symptoms and histological confirmation of eosinophile infiltration is necessary. Some investigators have claimed that a lack of response to proton pump inhibitors (PPIs) is necessary for a diagnosis of EoE, and patients who respond to such treatment are referred to as PPI-responsive esophageal eosinophilia (PPI-REE).

According to consensus recommendations published in 2011, the presence of esophageal symptoms, histological confirmation of esophageal mucosal eosinophile infiltration, and absence of pathological gastro-esophageal reflux or unresponsiveness to PPI treatment are all necessary factors for the diagnosis of EoE [20]. PPI-REE cases are considered to be separate from those with EoE in these recommendations. However, there are strong similarities in clinical and histo-pathological characteristics, and also gene expression patterns between EoE and PPI-REE [21-23]. In addition, PPIs were recently reported to have immuno-suppressive actions [24]. Therefore, it is possible that EoE and PPI-REE may be diseases with a common pathogenesis and need not be separated. Indeed, differential diagnosis between them is often difficult, and there is no consensus concerning 
necessary PPI doses or treatment length to distinguish them.

As a future diagnostic method, systematic analysis of mRNA expressed in esophageal mucosa may provide important findings. Blanchard et al. [25] performed microarray analysis of mRNA expressed in esophageal mucosa obtained from pediatric patients with EoE and found that the expressions of several cytokines, including eotaxin 3 and IL-13, were augmented, while those of filaggrin and involucrin were decreased. We also investigated mRNA expression in specimens obtained by endoscopic biopsy from Japanese adults with EoE and found similar alterations in gene expression [26]. Therefore, the pathogenetic mechanisms of EoE in Western and Asian patients are considered to be similar. Recently, Wen et al. [27] reported that the determination of the expression of 94 important genes in biopsy samples to characterize EoE was fast and objective as compared to a simple histological diagnosis method. A diagnostic panel of esophageal mucosal mRNA expression may become widely used for diagnosis in the near future. The current possible diagnostic algorism for patients with esophageal symptoms is shown as figure 3.

\section{Treatment}

EoE is a disease caused by food-induced allergic reactions in esophageal mucosa. Therefore, dietary and immuno-suppressive therapies are considered to be the main treatment options.

\section{Elemental Diets}

Elemental diets, developed and widely used for treatment of Crohn's disease, contain amino acids instead of protein and do not include food allergens. Therefore, consumption of such a diet without supplementation with conventional foods is a theoretically ideal treatment option for food allergen-induced diseases. In published reports, properly administered elemental diets were shown to be effective for many patients with EoE and can eliminate esophageal eosinophile infiltration within 1 month [28, 29]. Of the various dietary treatment options available, an elemental diet is one of the most effective, though patient adherence can be a problem because of unpleasant taste.

\section{Elimination Diets}

The six food elimination diet, which excludes wheat, milk, egg, nuts, soy, and fish/shell fish, is the most frequently employed dietary therapy especially for pediatric patients in Western countries, though it has also been reported to be effective for adult patients [30,31]. However,

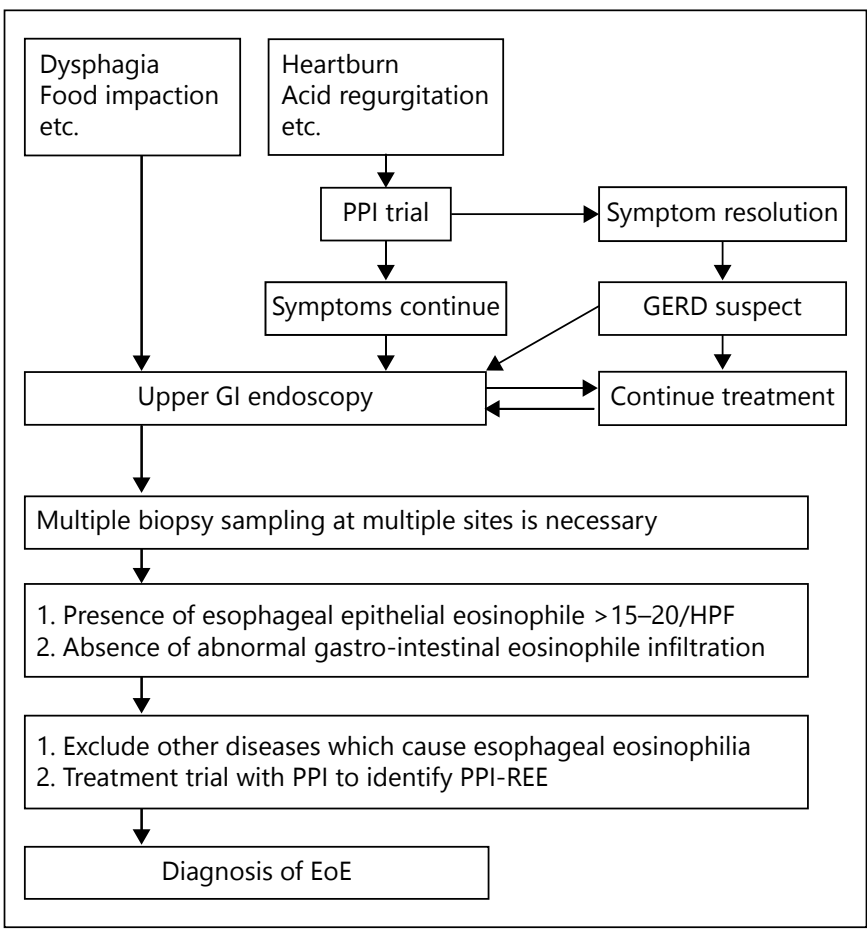

Fig. 3. Suggested diagnostic flowchart for EoE.

difficulty with compliance with this diet in working communities limits its effectiveness for adult patients. Nevertheless, approximately $70 \%$ of treated cases showed disappearance of eosinophile infiltration. To increase compliance to such dietary therapy, the four food elimination diet was developed [30]. Although reported to be effective, treatment success is lower than with the six food elimination diet.

When a patient shows response to an elimination diet, re-administration of the eliminated foods one by one must be done to identify the food allergen. Once identified, simple elimination of the allergen food is considered to be effective and adequate. The most frequently identified food allergens for adult patients with EoE are reported to be wheat and milk [32].

\section{Administration of PPIs}

PPIs may relieve EoE through both acid-related and -unrelated pathways [33]. Even in normal individuals, gastric acid frequently refluxes into the esophagus, with the lower esophagus exposed to acid for a period of up to $1 \mathrm{~h}$ each day. Such reflux is thought to damage esophageal squamous epithelium and facilitate allergen penetration deep into esophageal mucosa, thus increasing allergen exposure by the immune system and inducing or aggravat- 
ing EoE. Administration of acid inhibitors including PPIs is performed to strengthen the barrier function of esophageal mucosa and decrease allergen exposure. In addition to an effect on acid secretion, PPIs have also been reported to have an immune-suppressive effect especially on Th2 type responses. PPI administration directly suppresses IL-4 and IL-13-induced production of eotaxin 3 [24]. In Japan, more than half of patients with histologically confirmed EoE respond to PPI administration [34, 35]. Thus, PPIs are generally the first therapy chosen for cases with esophageal eosinophile infiltration, especially when considering the lower level of adverse effects.

\section{Immunosuppressant Treatment}

Glucocorticoid administration is the standard treatment option for EoE, with ingestion of a locally active agent such as fluticasone and budesonide an effective option. However, interruption of therapy even after attaining remission may aggravate disease activity and cause exacerbation. Oral candidiasis is also an adverse effect of local glucocorticoid ingestion therapy. The effectiveness of monoclonal antibody administration targeting tumor necrosis factor- $\alpha$, IgE, and IL- 5 and IL- 13 has been examined, and IL-5 and IL-13 neutralizing antibodies were found to have some beneficial effects [36-39], with the effect of the IL-13 antibody shown to be superior.

\section{Recent Progress in Basic and Clinical Research of EGE}

\section{Pathogenesis and Diagnosis}

Studies of the pathogenesis of EGE and diagnosis are limited. The condition is also considered to be a non-IgEdependent Th2 type allergic disease [40]. As with EoE, food allergens are believed to be the triggering and aggravating factors. For diagnosis of EGE, peripheral eosinophilia is frequently reported and its presence noted in 70$80 \%$ of confirmed cases [2]. The most frequently reported symptoms are abdominal pain and diarrhea, though the types of reported symptoms depend on the site of involvement [2]. Endoscopy has a limited role to detect the possible presence of EGE, since typical endoscopic abnormalities that are found are not specific, such as erosion, erythema, and edema. Therefore, a biopsy-based histopathological diagnosis is critically important. We recently determined the number of eosinophiles that had infiltrated esophago-gastro-intestinal mucosa in normal subjects and found that physiological mucosal eosinophile infiltration differed significantly in different segments of the gastrointestinal tract, with the distal ileum and ascending co- lon showing dense infiltration even in normal conditions [41]. For accurate diagnosis of EGE, different cutoff values should be established to discriminate the pathological from physiological eosinophile infiltration.

\section{Treatment}

No prospective randomized placebo controlled study of patients with EGE has been performed and evidencebased information concerning treatment options is limited. Glucocorticoid hormone administration is most frequently used and one-third of treated patients show a favorable response, including cure [42]. However, another one-third shows only a temporal response and then recurrence of disease activity during treatment withdrawal, and the other one-third has no response. For patients with EGE who show resistance to glucocorticoid administration, there is no recommended second-line therapy with adequate clinical evidence, which is necessary in the future.

\section{Conclusion}

In this report, recent progress in basic and clinical research of EoE and EGE is summarized. As for EoE, on the one hand, a variety of studies have been performed and new findings collected. On the other hand, information concerning EGE is limited and additional evidence is needed to establish effective treatment options.

\section{Disclosure Statement}

The authors have no conflicts of interest to declare concerning the contents of this manuscript.

\section{References}

1 Arora AS, Yamazaki K: Eosinophilic esophagitis: asthma of the esophagus? Clin Gastroenterol Hepatol 2004;2:523-530.

2 Kinoshita Y, Furuta K, Ishimaura N, et al: Clinical characteristics of Japanese patients with eosinophilic esophagitis and eosinophilic gastroenteritis. J Gastroenterol 2013;48: 333-339.

3 Prasad GA, Alexander JA, Schleck CD, et al: Epidemiology of eosinophilic esophagitis over three decades in Olmsted County, Minnesota. Clin Gastroenterol Hepatol 2009; 7:10551061.

4 Fujishiro H, Amano Y, Kushiyama Y, et al: Eosinophilic esophagitis investigated by upper gastrointestinal endoscopy in Japanese patients. J Gastroenterol 2011;46:1142-1144. 
5 Ishimura N, Shimura S, Jiao D, et al: Clinical features of eosinophilic esophagitis: differences between Asian and Western populations. J Gastroenterol Hepatol 2015;30(suppl 1):7177.

6 Sherrill JD, Rothenberg ME: Genetic dissection of eosinophilic esophagitis provides insight into disease pathogenesis and treatment strategies. J Allergy Clin Immunol 2011;128: 23-32; quiz 33-34.

7 Liu AH: Hygiene theory and allergy and asthma prevention. Paediatr Perinat Epidemiol 2007;21(suppl 3):2-7.

8 Ishimura N, Furuta K, Sato S, et al: Limited role of allergy testing in patients with eosinophilic gastrointestinal disorders. J Gastroenterol Hepatol 2013;28:1306-1313.

9 Furuta K, Adachi K, Aimi M, et al: Case-control study of association of eosinophilic gastrointestinal disorders with Helicobacter pylori infection in Japan. J Clin Biochem Nutr 2013;53:60-62.

10 Dellon ES, Peery AF, Shaheen NJ, et al: Inverse association of esophageal eosinophilia with Helicobacter pylori based on analysis of a US pathology database. Gastroenterology 2011;141:1586-1592.

11 D’Elios MM, Codolo G, Amedei A, et al: Helicobacter pylori, asthma and allergy. FEMS Immunol Med Microbiol 2009;56:1-8.

12 Mohammadi M, Czinn S, Redline R, et al: Helicobacter-specific cell-mediated immune responses display a predominant Th1 phenotype and promote a delayed-type hypersensitivity response in the stomachs of mice. J Immunol 1996;156:4729-4738.

13 Engler DB, Reuter S, van Wijck Y, et al: Effective treatment of allergic airway inflammation with Helicobacter pylori immunomodulators requires BATF3-dependent dendritic cells and IL-10. Proc Natl Acad Sci U S A 2014;111: 11810-11815.

14 Arnold IC, Dehzad N, Reuter S, et al: Helicobacter pylori infection prevents allergic asthma in mouse models through the induction of regulatory T cells. J Clin Invest 2011;121: 3088-3093.

15 Shimura S, Ishimura N, Tanimura T, et al: Reliability of symptoms and endoscopic findings for diagnosis of esophageal eosinophilia in a Japanese population. Digestion 2014;90: 49-57.

16 Kinoshita Y, Furuta K, Ishimura N, et al: Elevated plasma cytokines in Japanese patients with eosinophilic esophagitis and gastroenteritis. Digestion 2012;86:238-243.

17 Dellon ES, Rusin S, Gebhart JH, et al: Utility of a noninvasive serum biomarker panel for diagnosis and monitoring of eosinophilic esophagitis: a prospective study. Am J Gastroenterol 2015;110:821-827.
18 Nielsen JA, Lager DJ, Lewin M, et al: The optimal number of biopsy fragments to establish a morphologic diagnosis of eosinophilic esophagitis. Am J Gastroenterol 2014;109:515-520.

19 Remedios M, Campbell C, Jones DM, et al: Eosinophilic esophagitis in adults: clinical, endoscopic, histologic findings, and response to treatment with fluticasone propionate. Gastrointest Endosc 2006;63:3-12.

20 Liacouras CA, Furuta GT, Hirano I, et al: Eosinophilic esophagitis: updated consensus recommendations for children and adults. J Allergy Clin Immunol 2011;128:3-20.e6; quiz 21-22.

21 Dranove JE, Horn DS, Davis MA, et al: Predictors of response to proton pump inhibitor therapy among children with significant esophageal eosinophilia. J Pediatr 2009;154: 96-100.

22 Dellon ES, Speck O, Woodward K, et al: Clinical and endoscopic characteristics do not reliably differentiate PPI-responsive esophageal eosinophilia and eosinophilic esophagitis in patients undergoing upper endoscopy: a prospective cohort study. Am J Gastroenterol 2013;108:1854-1860.

23 Wen T, Dellon ES, Moawad FJ, et al: Transcriptome analysis of proton pump inhibitorresponsive esophageal eosinophilia reveals proton pump inhibitor-reversible allergic inflammation. J Allergy Clin Immunol 2015; 135:187-197.

24 Cheng E, Zhang X, Huo X, et al: Omeprazole blocks eotaxin-3 expression by oesophageal squamous cells from patients with eosinophilic oesophagitis and GORD. Gut 2013;62: 824-832.

25 Blanchard C, Wang N, Stringer KF, et al: Eotaxin-3 and a uniquely conserved gene-expression profile in eosinophilic esophagitis. J Clin Invest 2006;116:536-547.

26 Shoda T, Morita H, Nomura I, et al: Comparison of gene expression profiles in eosinophilic esophagitis (EoE) between Japan and western countries. Allergol Int 2015;64:260-265.

27 Wen T, Stucke EM, Grotjan TM, et al: Molecular diagnosis of eosinophilic esophagitis by gene expression profiling. Gastroenterology 2013;145:1289-1299.

28 Peterson KA, Byrne KR, Vinson LA, et al: Elemental diet induces histologic response in adult eosinophilic esophagitis. Am J Gastroenterol 2013;108:759-766.

29 Markowitz JE, Spergel JM, Ruchelli E, et al: Elemental diet is an effective treatment for eosinophilic esophagitis in children and adolescents. Am J Gastroenterol 2003;98:777-782.

30 Wolf WA, Jerath MR, Sperry SL, et al: Dietary elimination therapy is an effective option for adults with eosinophilic esophagitis. Clin Gastroenterol Hepatol 2014;12:1272-1279.
31 Gonsalves N, Yang GY, Doerfler B, et al: Elimination diet effectively treats eosinophilic esophagitis in adults; food reintroduction identifies causative factors. Gastroenterology 2012;142:1451-1459.e1; quiz e14-e15.

32 Kagalwalla AF, Shah A, Li BU, et al: Identification of specific foods responsible for inflammation in children with eosinophilic esophagitis successfully treated with empiric elimination diet. J Pediatr Gastroenterol Nutr 2011;53:145-149.

33 van Rhijn BD, Weijenborg PW, Verheij J, et al: Proton pump inhibitors partially restore mucosal integrity in patients with proton pump inhibitor-responsive esophageal eosinophilia but not eosinophilic esophagitis. Clin Gastroenterol Hepatol 2014;12:1815-1823. e2.

34 Abe Y, Iijima K, Ohara S, et al: A Japanese case series of 12 patients with esophageal eosinophilia. J Gastroenterol 2011;46:25-30.

35 Vazquez-Elizondo G, Ngamruengphong S, Khrisna M, et al: The outcome of patients with oesophageal eosinophilic infiltration after an eight-week trial of a proton pump inhibitor. Aliment Pharmacol Ther 2013;38:1312-1319.

36 Straumann A, Bussmann C, Conus S, et al: Anti-TNF-alpha (infliximab) therapy for severe adult eosinophilic esophagitis. J Allergy Clin Immunol 2008;122:425-427.

37 Foster B, Foroughi S, Yin Y, et al: Effect of anti-IgE therapy on food allergen specific $\mathrm{T}$ cell responses in eosinophil associated gastrointestinal disorders. Clin Mol Allergy 2011;9: 7.

38 Spergel JM, Rothenberg ME, Collins $\mathrm{MH}$, et al: Reslizumab in children and adolescents with eosinophilic esophagitis: results of a double-blind, randomized, placebo-controlled trial. J Allergy Clin Immunol 2012;129: 456-463, 463.e1-e3.

39 Rothenberg ME, Wen T, Greenberg A, et al: Intravenous anti-IL-13 mAb QAX576 for the treatment of eosinophilic esophagitis. J Allergy Clin Immunol 2015;135:500-507.

40 Caldwell JM, Collins MH, Stucke EM, et al: Histologic eosinophilic gastritis is a systemic disorder associated with blood and extragastric eosinophilia, $\mathrm{TH} 2$ immunity, and a unique gastric transcriptome. J Allergy Clin Immunol 2014;134:1114-1124.

41 Matsushita T, Maruyama R, Ishikawa N, et al: The number and distribution of eosinophils in the adult human gastrointestinal tract: a study and comparison of racial and environmental factors. Am J Surg Pathol 2015;39: 521-527.

42 Pineton de Chambrun G, Gonzalez F, Canva JY, et al: Natural history of eosinophilic gastroenteritis. Clin Gastroenterol Hepatol 2011; 9:950-956.e1. 